\title{
Neumonía eosinofílica crónica en un paciente con artritis reumatoide en tratamiento con sulfasalazina
}

\section{Chronic eosinophilic pneumonia in a patient with rheumatoid arthritis on treatment with sulfasalazine}

Luis Fernando Guerrero González(1); Guillermo Garrido(2); Paulina Ojeda León ${ }^{(3)}$

\begin{abstract}
RESUMEN
La neumonía eosinofílica crónica es una enfermedad poco conocida, caracterizada por un deterioro respiratorio progresivo mayor de un mes de evolución, con presencia de infiltrados pulmonares y eosinofília. Se han reportado varios casos de asociación con sulfasalazina en enfermedad inflamatoria intestinal. Describimos un caso de neumonía eosinofílica crónica en un paciente con antecedente de artritis reumatoide en tratamiento con sulfasalazina.
\end{abstract}

Palabras clave: neumonía eosinofílica crónica, sulfasalazina, artritis reumatoidea.

\begin{abstract}
Chronic eosinophilic pneumonia is a scarcely known disease characterized by progressive respiratory deterioration evolving over more than one month, with presence of lung infiltrates and eosinophilia. Several cases associated with sulfasalazine used for inflammatory bowel disease have been reported. We describe a case of chronic eosinophilic pneumonia in a patient with rheumatoid arthritis on treatment with sulfasalazine.
\end{abstract}

Key words: chronic eosinophilic pneumonia, sulfasalazine, rheumatoid arthritis

Rev Colomb Neumol 2011; 23(1): 30-33.

\section{INTRODUCCIÓN}

Las neumonías eosinofílicas son caracterizadas por la presencia de tos, infiltrados pulmonares y eosinofília pulmonar. Existen dos clases de neumonía eosinofílica, aguda y crónica, diferenciadas porque la crónica tiene un inicio más insidioso, fiebre leve y menos hipoxia, que la forma aguda que generalmente se presenta durante los primeros 15 días de inicio del cuadro clínico (1).

La neumonía eosinofílica crónica es una enfermedad poco común, que se ha relacionado con varios factores, dentro de los cuales se encuentra la artritis reumatoide y el tratamiento con sulfasalazina en pacientes con enfermedad intestinal inflamatoria. Varios efectos adversos han sido descritos como náuseas, dolor abdominal, cefalea, anorexia, rash, infertilidad y síndromes pulmonares con el uso de esta medicación (16).

Describimos un caso de neumonía eosinofílica crónica en un paciente con artritis reumatoide y manejo con sulfasalazina durante tres años, el cual fue diagnosticado con biopsia pulmonar.

\section{REPORTE DE CASO}

Paciente masculino de 71 años de raza blanca quien labora en un depósito de residuos industriales. Consulta por cuadro clínico de 2 meses consistente en tos con expectoración blanca y disnea progresiva. Anteceden-

\footnotetext{
MD. Residente de 2do año de Medicina Interna, Universidad Libre. Cali, Colombia

MD. Neumólogo, Corporación Comfenalco - Universidad Libre. Docente, Universidad Libre. Cali, Colombia.

MD. Patóloga pulmonar, Hospital Santa Clara. Bogotá, Colombia.
}

Correspondencia: Doctor Luis Fernando Guerrero González, correo electrónico: luisguerrero81@yahoo.es

Recibido: marzo 15 de 2011. Aceptado: marzo 22 de 2011. 
tes de hipertensión arterial controlada con losartan 75mg/ día, glaucoma, hernia discal y artritis reumatoide diagnosticada hace 3 años, por lo cual recibía manejo con sulfasalazina, inicialmente 1,5mg/día con disminución en el último año a $500 \mathrm{mg} /$ día. Al examen físico se encuentra hemodinámicamente estable, sin signos de dificultad respiratoria y presencia de estertores basales. Se realiza radiografía de tórax (Figura 1) y TAC de tórax (Figuras 2 y 3) que muestran infiltrados en parches y áreas de vidrio esmerilado periférico. factor reumatoideo $79 \mathrm{U} / \mathrm{ml}$ (positivo), ANAs negativo, velocidad de eritrosedimentación de $57 \mathrm{~mm} / \mathrm{hr}$. Hemograma mostraba leucocitos: 18.590 , neutrófilos: $51 \%$, linfocitos: $12 \%$, eosinófilos: $26,7 \%(4,970 / \mathrm{mm} 3), \mathrm{Hb}: 13,2 \mathrm{gr} / \mathrm{dl}$, plaquetas: 452.000 .

El paciente fue hospitalizado por persistencia y empeoramiento de los síntomas y durante su estancia hospitalaria se realizó biopsia pulmonar, la cual reportó neumonía eosinofílica (Figuras 4 y 5).

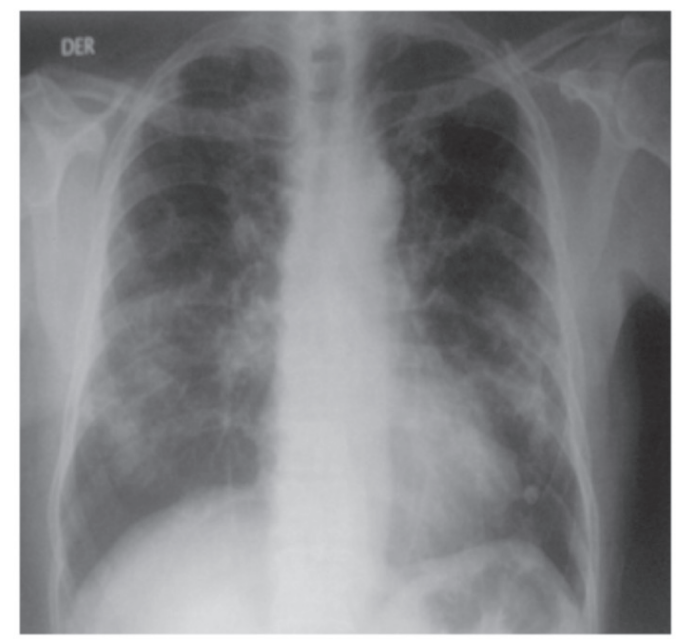

Figura 1. Radiografía de tórax. Infiltrados intersticiales periféricos.

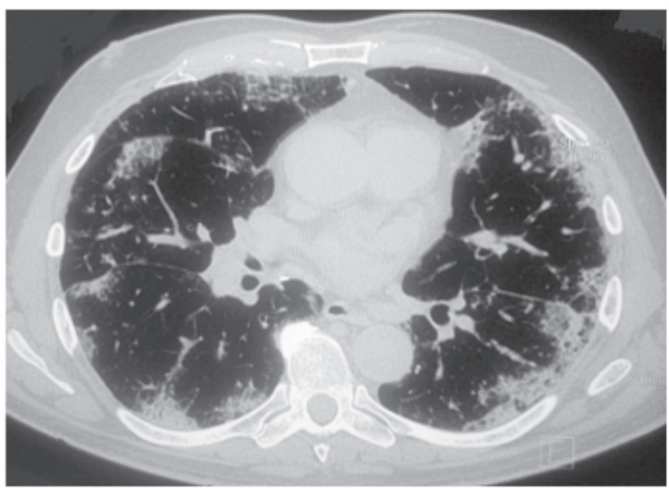

Figura 2. TAC de tórax.
Con el diagnóstico histopatológico se inició manejo con metilprednisolona $32 \mathrm{mg} /$ día durante 2 semanas, con disminución progresiva de la dosis previa cada dos semanas, hasta suspenderla a los dos meses. Luego de terminar tratamiento con el corticoesteroide, se realiza radiografía de tórax de control, la cual evidencia mejoría de infiltrados. La espirometría un mes posterior fue normal. El paciente informa mejoría clínica luego del tratamiento con corticoesteroides.

\section{DISCUSIÓN}

La neumonía eosinofílica crónica es una enfermedad de causa desconocida, más común en mujeres y que usualmente se presenta después de la cuarta década de vida, sin embargo, se han descrito algunos casos entre los 15 y 80 años de edad $(2,3)$.

Aunque muchos pacientes son sanos antes del inicio de la enfermedad, se ha descrito que $50 \%$ a $62 \%$ de los pacientes tienen asma o atopía $(4,14)$. Algunos casos se han producido en asociación con diferentes entidades, como la artritis reumatoide $(1,5,6)$, radioterapia para el cáncer de mama (7), y larva migrans visceral (8). Menos del $10 \%$ de los casos son fumadores activos (2, $3,9)$.

La neumonía eosinofílica crónica puede ser causada por diferentes medicamentos. Los medicamentos más frecuentes asociados son los antibióticos incluyendo a la minociclina, la nitrofurantoina y la sulfasalazina $(15,16)$. La prescripción de sulfasalazina se ha incrementado en los últimos años y se han reportado varios casos asociados con la toxicidad pulmonar, por lo cual debe considerarse en el diagnóstico diferencial de enfermedad pulmonar intersticial. La mayoría de los pacientes con sospecha de neumonía eosinofílica crónica inducida por sulfasalazina mejoran con la suspensión del medicamento y el uso de corticoesteroides (15).

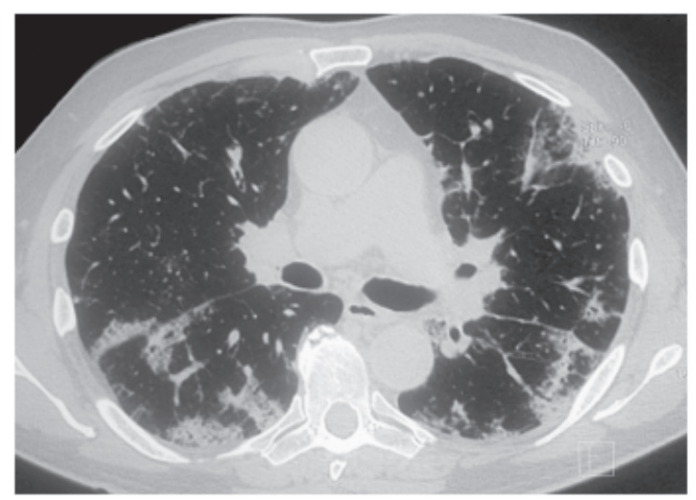

Figura 3. TAC de tórax. 
Dentro de la presentación clínica, la tos es el síntoma más común (presentado en más de $93 \%$ de los casos), fiebre (77\%-87\%), disnea (57\%-92\%), pérdida de peso (57\%-75\%), diaforesis nocturna (23\%) (2).

Las anormalidades de laboratorio más comunes son la eosinofília, puede encontrarse elevación de las plaquetas, reactantes de fase aguda (VES) elevados, factor reumatoideo y circulación de complejos inmunes. En las pruebas de función pulmonar pueden encontrarse disminución de la difusión de monóxido de carbono, patrón obstructivo o restrictivo en las espirometrías (13). La fibrobroncoscopia y la biopsia pulmonar no son siempre requeridas. Si la fibrobroncoscopia es realizada, el lavado bronquioalveolar (LBA) evidenciará un conteo elevado de eosinófilos (10). Los hallazgos más frecuentes

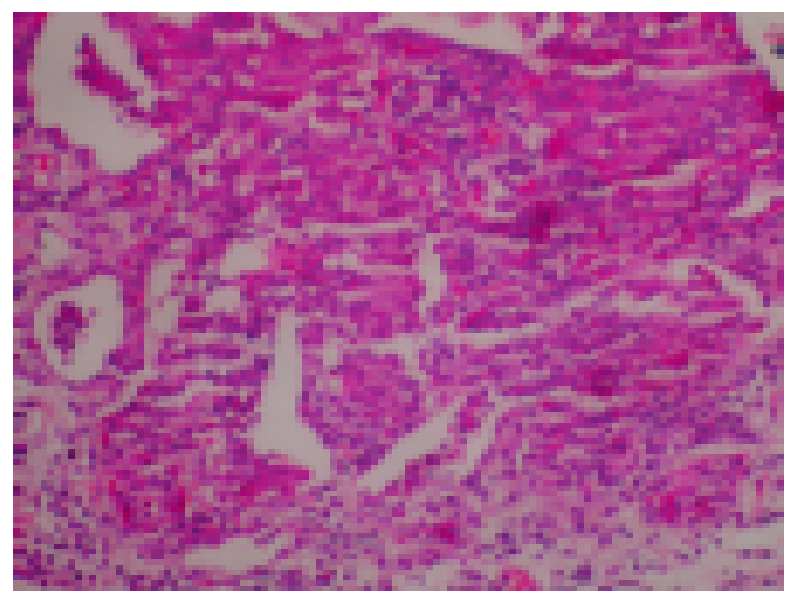

Figura 4 .Ocupación de la luz de los alveolos por histiocitos y eosinófilos. HE 10X.

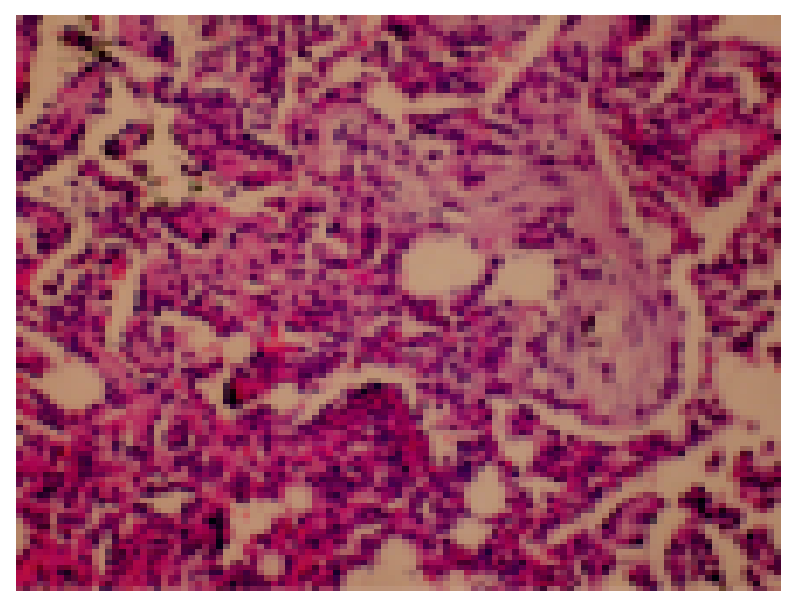

Figura 5. Presencia de pólipo fibroblástico en la luz de un bronquiolo y alveolo (cambio de BOOP focal). HE 10X. en la radiografía de tórax son infiltrados bilaterales de predominio periférico, sin embargo, se han reportado hallazgos atípicos radiográficos como consolidaciones, cavitaciones, derrame pleural, infiltrados nodulares y atelectasias (10).

El diagnóstico es usualmente basado en la asociación de (11):

1. Síntomas respiratorios usualmente mayores a 2 semanas de duración.

2. Eosinofilia sanguínea y/o alveolar (eosinofília alveolar $>40 \%$ en el LBA, eosinofília sanguínea $>1000 / \mathrm{mm}^{3}$ )

3. Infiltrados pulmonares con predominio periférico en la radiografía de tórax.

4. Exclusión de alguna causa conocida de enfermedad pulmonar eosinofílica.

El pilar del tratamiento son los corticoesteroides, generalmente puede iniciarse una dosis de $60 \mathrm{mg} /$ día de prednisolona, aunque algunos estudios reportan dosis de 0,3 a $3 \mathrm{mg} / \mathrm{kg} / \mathrm{día}$ (9).Los corticoides inhalados no son efectivos como monoterapia (12). La respuesta terapéutica es usualmente reflejada dentro de las primeras dos semanas y debe continuarse por un largo periodo. Las recaídas son comunes cuando el tratamiento con los corticoesteroides es suspendido o disminuido $(3,9)$. La mortalidad es poco común pero se ha reportado.

En el caso descrito, el paciente presentaba una enfermedad crónica que se ha asociado a la aparición de neumonía eosinofílica crónica, sin embargo, estuvo en tratamiento con sulfasalazina que igualmente desencadena toxicidad pulmonar y que podría desencadenar la enfermedad. Por tal razón, se concluye en este caso, que probablemente las dos entidades descritas, artritis reumatoide y sulfasalazina, estén asociadas con el inicio de neumonía eosinofílica crónica.

\section{REFERENCIAS}

1. Norman D, Piecyk M, Roberts DH. Eosinophilic pneumonia as an initial manifestation of rheumatoid arthritis. Chest 2004; 126(3): 993-5.

2. Jederlinic PJ, Sicilian L, Gaensler EA. Chronic eosinophilic pneumonia: a report of 19 cases and a review of the literature. Medicine (Baltimore) 1988; 67(3): 154-62.

3. Naughton M, Fahy J, FitzGerald MX. Chronic eosinophilic pneumonia: a long- term follow-up of 12 patients. Chest 1993; 103(1): 162-5.

4. Pope-Harman AL, Davis WB, Allen ED, Christoforidis AJ, Allen JN. Acute eosinophilic pneumonia. A summary of 15 cases and review of the literature. Medicine 1996, 75: 334-42.

5. Connellan s.j, Payne C. Chronic eosinophilic pneumonia complicating long- standing rheumatoid arthritis. Postgraduate Medical Journal 1980; 56: 519-20. 
6. Cooney TP. Interrelationship of chronic eosinophilic pneumonia, bronchiolitis obliterans, and rheumatoid disease: a hypothesis. J Clin Pathol 1981; 34: 129-37.

7. Cottin V, Frognier R, Monnot H, Levy A, DeVuyst P, Cordier JF; Groupe d'Etudes et de Recherche sur les Maladies "Orphelines" Pulmonaires. Chronic eosinophilic pneumonia after radiation therapy for breast cancer. Eur Respir J 2004; 23(1): 9-13.

8. Inoue $\mathrm{K}$, Inoue $\mathrm{Y}$, Arai T, Nawa Y, Kashiwa Y, Yamamoto S,Sakatani M. Chronic eosinophilic pneumonia due to visceral larva migrans. Intern Med 2002; 41(6): 478-82.

9. Marchand E, Reynaud-Gaubert M, Lauque D, Durieu J, Tonnel $\mathrm{AB}$, Cordier JF. Idiopathic chronic eosinophilic pneumonia: a clinical and follow-up study of 62 cases. The Groupe d'Etudes et de Recherche sur les Maladies "Orphelines" Pulmonaires (GERM"O"P). Medicine (Baltimore) 1998; 77(5): 299-312.

10. Luks AM, Altemeier WA. Typical Symptoms and Atypical Radiographic Findings in a Case of Chronic Eosinophilic Pneumonia. Respiratory care, 2006; 51(7): 764-7.
11. Marchand E, Cordier JF. Idiopathic chronic eosinophilic pneumonia. Orphanet Journal of Rare Diseases 2006; 1: 11.

12. Minakuchi M, Niimi A, Matsumoto H, Amitani R, Mishima M. Chronic eosinophilic pneumonia: treatment with inhaled corticosteroids. Respiration 2003; 70(4): 362-66.

13. Durieu J, Wallaert B,Tonnel A, and the Groupe d'Etude en Pathologie Interstitielle de la Société de Pathologie Thoracique du Nord. Long-term follow-up of pulmonary function in chronic eosinophilic Pneumonia. Eur Respir J 1997; 10: 286-91.

14. Marchand E, Etienne-Mastroianni B, Chanez P, Lauque L, Leclerc P, Cordier JF, and the Groupe d'Etudes et de Recherche sur les Maladies Orphelines Pulmonaires. Idiopathic chronic eosinophilic pneumonia and asthma: how do they influence each other? Eur Respir J 2003; 22: 8-13.

15. Parry S, Barbatzas C, Peel E, Barton R. Sulphasalazine and lung toxicity. Eur Respir J 2002; 19: 756-64.

16. Tanigawa K, Sugiyama K, Matsuyama H, Nakao H, et al. Mesalazine-Induced Eosinophilic Pneumonia. Respiration 1999; 66: 69-72. 